\title{
Correction to: Repositioning Pedagogical Content Knowledge in Teachers' Knowledge for Teaching Science
}

\author{
Anne Hume, Rebecca Cooper, and Andreas Borowski
}

\section{Correction to:}

\author{
A. Hume et al. (eds.), \\ Repositioning Pedagogical Content Knowledge in Teachers' \\ Knowledge for Teaching Science, \\ https://doi.org/10.1007/978-981-13-5898-2
}

The original version of the book was inadvertently published with incorrect information in chapter 2, chapter 12 and in backmatter. The erratum book has been updated with the changes. 